Also, it is apparent that the gap in generating evidence and practicing medicine is getting wider to cover, as most of the efforts are put into trials to establish superiority of one method over another rather than improving quality of care by striving to incorporate it in daily practice. 1

The main objectives are to assess the obstacles in the process of incorporation of EBM in day to day practice starting from lack of realisation of right question to challenge old and outdated practice to the development of strong evidence and implementation in order to improve clinical practice.

Method A case base discussion was performed with Trainees and Special Doctors in the field of Urology.

The literature search was performed on Cochrane, Pubmed and Google scholar to identify the role of leadership in EBM and accessibiltiy of under training doctors to understand, comprehend and implement the need of practicing Evidence Based Medicine.

Results The factors that have been found closely related to understand the role of health care personnel in EBM are Importance of realising the incorporation of EBM in day to day practice, The Role of health care personnel in generating relevant evidence, Structure of training of health personnel, Factors hampering the evidence synthesis, Choice of Evidence synthesis, role of clinical expertise and Leaders in Evidence Based medicine.

Conclusions The role models in EBM will require to get trained to be enlightened by the importance of EBM. The aim of bringing the clinicians on a platform to share a common goal of improvement in clinical practice, where they can address issues of obstacles in data acquisition, dissemination and implementation of evidence proven practice, administrative support provision as well as advances in the methods of improved accessibility of EBM.

\section{THE CREATION OF THE OXFORD-BRAZIL EBM ALLIANCE}

1,2,3 Luis Fontes, ${ }^{4,5}$ Rachel Riera, ${ }^{1,6}$ Regis Vieira, ${ }^{1,7}$ Rafael Pacheco, ${ }^{1,8}$ Ana Luiza Martimbianco, ${ }^{1,9}$ Daniela Pachito, ${ }^{10}$ Enderson Miranda, ${ }^{11}$ David Nunan. ${ }^{1}$ Universidade Federal de São Paulo (UNIFESP), São Paulo, Brazil; ${ }^{2}$ Faculdade de Medicina de Petrópolis, Petrópolis, Brazil; ${ }^{3}$ Department for Continuing Education, University of Oxford, Oxford, UK; ${ }^{4}$ Escola Paulista de Medicina (EPM) at Universidade Federal de São Paulo (UNIFESP), São Paulo, Brazil; ${ }^{5}$ Center of Health Technology Assessment, Hospital Sírio-Libanês, São Paulo, Brazil; ${ }^{6}$ Hospital Israelita Albert Einstein, São Paulo, Brazil; ${ }^{7}$ Centro Universitário São Camilo (CUSC), São Paulo, Brazil; ${ }^{8}$ Universidade Metropolitana de Santos, Santos, Brazil; ${ }^{9}$ Gestão e Economia de Saúde; Hospital Sírio-Libanês, São Paulo, São Paulo, Brazil; ${ }^{10}$ Centre for Evidence Based Medicine (CEBM), Department for Continuing Education, University of Oxford, Oxford, UK; ${ }^{11}$ Centre for Evidence Based Medicine (CEBM), Nuffield Department of Primary Care, Health Sciences, University of Oxford, Oxford, UK

\subsection{6/bmjebm-2019-EBMLive.96}

Objectives To describe the experience of building a teaching partnership between the Oxford CEBM and leading Brazilian Medical Schools, thus aiming to increase the effectiveness and quality of EBM teaching in Brazil.

Brazil is the 5th largest country in the world, with a population of approximately 210 million people. There are 336 active medical schools in Brazil, which graduate an average of 34,000 medical professionals each year. Of these schools, less than 20\% have in their curricula a formal Evidence-Based Medicine (EBM) discipline. The lack of formal EBM integration into medical school curricula is not exclusively a national challenge for Brazil. Data from the USA and UK suggests that only $60 \%$ of curricula include formal EBM content. Despite the recognition of the importance of EBM education and its influence on medical training around the world, challenges remain in integrating EBM education into the medical curriculum, facilitating communication between practitioners, increasing the capacity for EBM's meaningful use in clinical practice, and ensuring medical professionals have the knowledge and skills to support evidence-based practice. Concerned about these issues, two Oxford post-graduate students of EvidenceBased Health Care and a Senior Research Fellow of the Oxford Centre for Evidence-Based Medicine (CEBM) proposed the creation of a partnership between Oxford's CEBM and the EBM Departments of leading Brazilian medical schools. This partnership, named the 'Oxford-Brazil EBM Alliance,' aims to provide Evidence-Based Medicine and curriculum development training to medical educators (formal professors or tutors) in Brazil, thus fostering sustainability and replicability of effective teaching and learning throughout medical schools in Brazil. Objective: To describe the experience of building a teaching partnership between the Oxford CEBM and leading Brazilian Medical Schools, thus aiming to increase the effectiveness and quality of EBM teaching in Brazil. Method: Descriptive Case Study. Results: Two prominent Brazilian medical schools already joined the Oxford-Brazil EBM Alliance: Escola Paulista de Medicina at Universidade Federal de São Paulo (EPM-UNIFESP) and Faculdade de Medicina de Petrópolis (FMP-FASE). Training will be delivered via 3-day workshops, starting in June 2019, which will be facilitated by faculty from Oxford and Brazil. Other activities may emerge afterwards as instigated by local institutions. This project is underpinned in a concept of developing a 'Center of Excellence in EBM Teaching and Training', which intends to set the standard for EBM teaching in Brazil and throughout South America. It is our overarching aim that over time Brazil will have qualified faculty members to teach EBM in all its Medical Teaching Institutions. To our knowledge, a similar partnership does not exist in our region. In a second phase, we intend to extend this project to other countries in South America, offering further opportunities for international collaboration.

Method Descriptive Case Study.

Results Two prominent Brazilian medical schools already joined the Oxford-Brazil EBM Alliance: Escola Paulista de Medicina at Universidade Federal de São Paulo (EPM-UNIFESP) and Faculdade de Medicina de Petrópolis (FMP-FASE). Training will be delivered via 3-day workshops, starting in June 2019, which will be facilitated by faculty from Oxford and Brazil. Other activities may emerge afterwards as instigated by local institutions. This project is underpinned in a concept of developing a 'Center of Excellence in EBM Teaching and Training', which intends to set the standard for EBM teaching in Brazil and throughout South America. It is our overarching aim that over time Brazil will have qualified faculty members to teach EBM in all its Medical Teaching Institutions. To our knowledge, a similar partnership does not exist in our region. In a second phase, we intend to extend this project to other countries in South America, offering further opportunities for international collaboration.

Conclusions The Oxford - Brazil EBM Alliance is a promising partnership that can bring Evidence-Based Medicine training to thousands of medical professors and interdisciplinary health care teams throughout Brazil and its neighboring countries. The proposed 'teach the teacher' approach can facilitate EBM teaching and learning, foster future leaders, and ultimately benefit patients and healthcare systems throughout Brazil. 